\title{
Case Control Study on Dialysis Arthropathy: The Influence of Two Different Dialysis Membranes: Data from the EDTA Registry
}

\author{
F. P. Brunner* Departement für Innere Medizin, \\ Universität Basel, Switzerland \\ H. Brynger Department of Surgery 1, Sahlgrenska \\ Sjukhuset, Göteborg, Sweden \\ J. H. H. Ehrich Medizinische Hochschule Hannover, \\ Federal Republic of Germany \\ W. Fassbinder Städtische Kliniken Fulda, Federal \\ Republic of Germany
}

W. Geerlings

G. Rizzoni

N. H. Selwood

G. Tufveson

A. J. Wing
Stichting Thuisdialyse Noord Nederland, Haren, Netherlands Ospedale Pediatrico Bambino Gesú, Istituto di Ricerca Scientifica, Roma, Italy UK Transplant Service, Bristol, United Kingdom Transplantation Unit, University Hospital, Uppsala, Sweden
St Thomas' Hospital, London, United Kingdom

*Chairman

\begin{abstract}
In a retrospective case control study the prevalence of signs and symptoms of dialysis osteoarthropathy was analysed. Cases and controls had received over 9 years of maintenance haemodialysis uninterrupted by peritoneal dialysis or transplantation. The cases comprised 55 patients treated predominantly with polyacrylonitrile (AN69) dialysers. They were compared to a matched group dialysed exclusively with cellulosic membranes. Over $60 \%$ of all patients, cases and controls, showed one or more signs of disabling osteoarthropathy, with joint pains occurring more frequently in the older age groups. Twenty-seven of the 55 cases who had received less than 2 years of cellulosic membrane dialysis followed by 7-12 years of AN69 dialysis tended to have a lower prevalence of joint pains, carpal-tunnel syndrome and bone cysts. However, no statistically significant differences were obtained compared to the matched control
\end{abstract}

Correspondence and offprint requests to: Professor F. P. Brunner, EDTA Registry, St Thomas's Hospital, London SE1 7EH, UK. group dialysed exclusively on cellulosic membranes (mostly cuprophane). The remaining 28 cases, who had been treated for more than 2 years with cellulosic membranes preceding the longer treatment period with polyacrylonitrile dialysers, showed a prevalence similar to that of their cellulosic controls.

This study thus shows little, if any, influence of the two types of membranes on the prevalence of signs and symptoms of $\beta_{2}$-microglobulin amyloidosis.

Key words: $\beta_{2}$-microglobulin amyloidosis; Case-control study; Cellulosic membranes; AN69; EDTA Registry

\section{Introduction}

Dialysis arthropathy has been recognised in recent years as a severe and disabling condition with signs and symptoms developing after some 10 years on haemodialysis. This new type of arthropathy is frequently accompanied 
by carpal-tunnel syndrome (CTS) and periarticular cysts can be observed on X-ray examination. The full-blown disease is characterised by joint pains and erosive polyarthropathy as well as CTS and periarticular cysts. Histological examination of synovia and/or bone cysts usually reveals the presence of a special type of amyloid, namely $\beta_{2}$-microglobulin [1-6]. $\beta_{2}$-Microglobulin is a single polypeptide chain with a molecular weight of 11800 daltons, which is eliminated almost entirely through the kidney. In patients on haemodialysis, blood concentrations are markedly increased although this may be influenced by residual renal function, number of years on haemodialysis and type of membrane used for haemodialysis.

The haemodialysis membrane polyacrylonitrile (AN69) is well known for some very specific properties, including its capacity to remove $\beta_{2}$-microglobulin. It has been claimed by some authors that no signs of haemodialysisassociated amyloidosis could be found in patients who had been dialysed for up to 10 years solely with this membrane $[7,8]$. The possible relationship between types of membrane and prevalence of this newly described disease is difficult to evaluate in clinical research, as numbers of comparable haemodialysis patients, treated for more than 10 years and in whom the major discriminant is the dialysis membrane, are small.

The Registry of the European Dialysis and Transplant Association-European Renal Association (EDTA Registry) designed a retrospective case control study to investigate the prevalence of dialysis arthropathy in two groups of patients. The major difference between these two groups was the use of two different dialysis membranes, namely a cellulosic membrane (mostly cuprophane) and polyacrylonitrile (all AN69). Using the EDTA Registry database of approximately 230000 patient records, patients were identified who had been treated by haemodialysis without interruption for 9 or more years and who had used predominantly either AN69 or cuprophane membranes. A special questionnaire concerning signs and symptoms of the disease was designed and sent in late 1986 and in early 1987 to those centres responsible for the care of these patients.

\section{Materials and Methods}

The cases were selected with the following inclusion criteria:

1. The cause of end-stage renal failure had to be a standard primary renal disease (glomerulonephritis, pyelonephritis/interstitial nephritis, toxic nephropathy, cystic renal diseases, chronic renal failure of uncertain aetiology). Thus patients with systemic diseases, diabetic nephropathy, amyloidosis and myeloma, as well as carcinoma, were excluded.
2. Haemodialysis, started in $\mathbf{1 9 7 6}$ or earlier, had to be the sole method of treatment from the start of renal replacement therapy (RRT). The treatment sequence had not to be interrupted by transplantation, CAPD or recovery of renal function until 31 December 1985.

3. Patients had to be registered and alive on 31 December 1985.

4. The cases had to be on polyacrylonitrile (AN69) dialysers starting in 1981 or earlier.

A total of 296 questionnaires were sent out for patients who met the first three of these inclusion criteria and for whom an AN69 dialyser was recorded as the most commonly used dialyser in 1985. Manual checking of the returns allowed the identification of 81 cases who had been treated with AN69 dialysers since 1981 or earlier. For each AN69 case, a control patient was selected from the EDTA Registry file using Registry software developed for this purpose. Cases and controls had to be of the same sex and come from the same country but the age of the control patient could vary \pm five years of that of the AN69 case. Potential control cases were listed and then an individual control case was selected by using a random number table. The mean number of controls for each case was 25 (median: 24) and varied between 1 and 50 . No control patient could be found for two of the 81 cases. Of the 79 control questionnaires that were sent out, 55 could be included. In 27 of the 55 AN69 cases, dialysis by AN69 had been preceded by less than 2 years and in 28 by more than 2 years of dialysis with a cellulosic membrane, mostly cuprophane, before switching to AN69. Statistical analysis was performed using the $\chi^{2}$ test with Pearson's correction and the rank sum test.

The questionnaire for each patient requested information on presence or absence of joint pains, evidence of CTS, radiological evidence of periarticular cystic bone lesions, radiological evidence of erosive polyarthropathy, histological evidence of amyloid and serum $\boldsymbol{\beta}_{2^{-}}$ microglobulin level if available. For each patient, data concerning start of treatment and type of membrane used had to be confirmed by the centre.

\section{Results}

Table 1 shows the patient characteristics of the two AN69 groups and their cuprophane controls. Median ages were matched, as were the number of years that patients had been treated by haemodialysis. The number of years that patients had been treated with AN69 or cuprophane is also given and a differentiation is made between those treated with cellulosic membranes for less than 2 years before switching to AN69 versus those previously treated for more than 2 years with cellulosic membrane. It should be noted that the number of years in Table 1 relate to 
Table 1. Median age and duration of uninterrupted maintenance haemodialysis on 31 December 1985 in two groups of patients with the membrane type (cellulosic vs AN69) as the most common discriminant

\begin{tabular}{|c|c|c|c|c|}
\hline & $\begin{array}{l}\text { AN69 } \\
<2 \text { years cellulosic } \\
\text { membrane } \\
(27 \text { cases) }\end{array}$ & $\begin{array}{l}\text { Cuprophane } \\
\text { ( } 27 \text { controls) }\end{array}$ & $\begin{array}{l}\text { AN69 } \\
>2 \text { years cellulosic } \\
\text { membrane } \\
\text { (28 cases) }\end{array}$ & $\begin{array}{l}\text { Cuprophane } \\
\text { ( } 28 \text { controls) }\end{array}$ \\
\hline Median age & 58 & 59 & 60 & 60 \\
\hline $\begin{array}{l}\text { Years on dialysis } \\
\text { (median range) }\end{array}$ & $10(9-12)$ & $10 \cdot(9-12)$ & $12(10-17)$ & $11(9-17)$ \\
\hline $\begin{array}{l}\text { Years on same membrane } \\
\text { (median range) }\end{array}$ & $10(7-12)$ & $10(7-12)$ & $7(4-11)$ & $11(9-17)$ \\
\hline
\end{tabular}

Table 2. Prevalence of signs and symptoms of dialysis osteoarthropathy after long-term haemodialysis in patients treated predominantly with AN69 or with cellulosic membranes

\begin{tabular}{|c|c|c|c|c|c|c|}
\hline & $\begin{array}{l}\text { AN69 } \\
\text { (<2 years } \\
\text { cell. membr.) }\end{array}$ & $P$ value & Controls & $\begin{array}{l}\text { AN69 } \\
(>2 \text { years } \\
\text { cell. membr.) }\end{array}$ & $P$ value & Controls \\
\hline Joint pains & $13 / 26$ & 0.17 & $18 / 26$ & $21 / 25$ & 0.71 & $20 / 25$ \\
\hline CTS & $7 / 26$ & 0.38 & $10 / 26$ & $11 / 27$ & 0.43 & $14 / 27$ \\
\hline Cysts & $6 / 26$ & 0.23 & $10 / 26$ & $10 / 27$ & 0.77 & $9 / 27$ \\
\hline Erosive arthropathy & $6 / 25$ & 0.89 & $6 / 24$ & $7 / 27$ & 0.15 & $12 / 27$ \\
\hline
\end{tabular}

Cases treated with cellulosic membranes for less than 2 years, and those treated for more than 2 years, before switching to AN69 are recorded separately

31 December 1985, whilst collection of clinical data by completing the questionnaires occurred 12-18 months later.

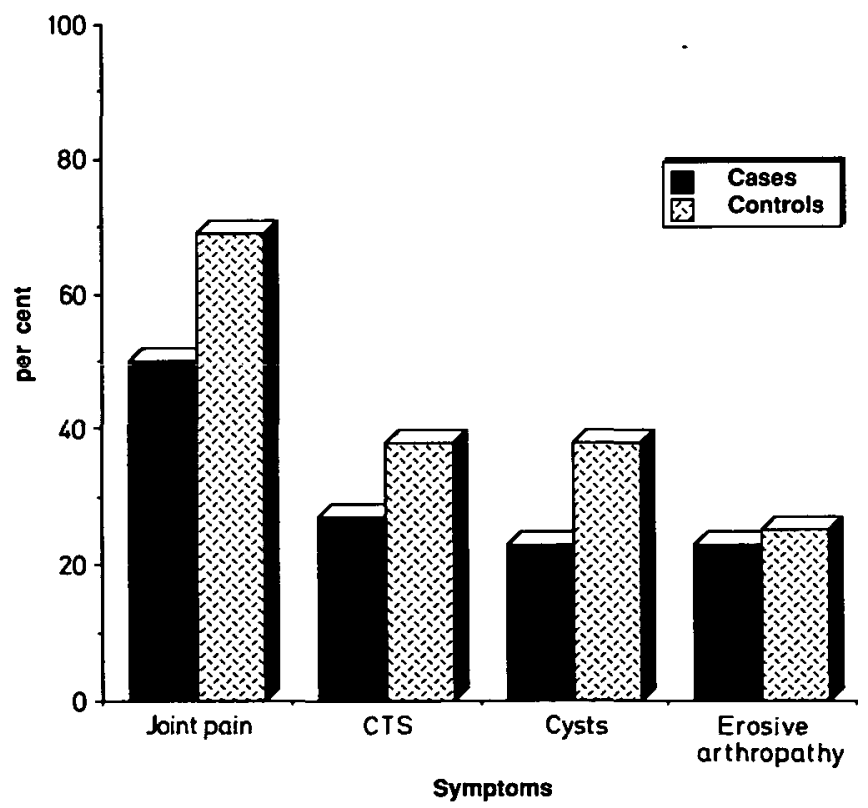

Fig. 1. Prevalence of signs and symptoms of dialysis osteoarthropathy in the 27 AN69 cases with less than 2 years haemodialysis on cellulosic membranes and their controls.

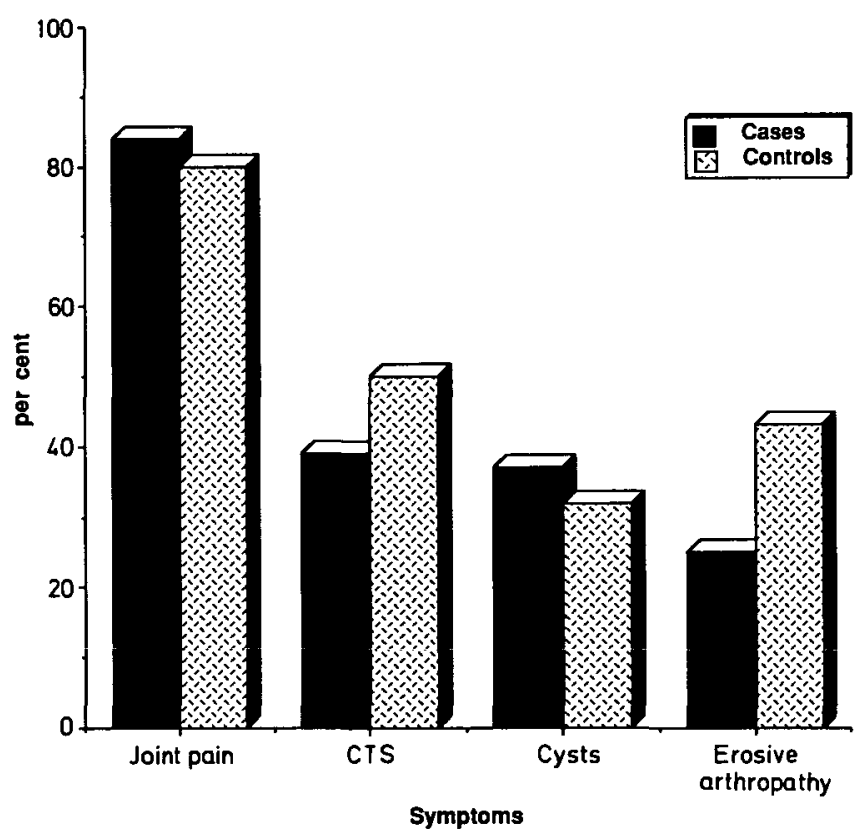

Fig. 2. Prevalence of signs and symptoms of dialysis osteoarthropathy in the 28 AN69 cases with more than 2 years haemodialysis on cellulosic membranes and their controls.

The prevalence of signs and symptoms of dialysisassociated amyloidosis is shown in Table 2 and Figs 1 and 2. Although, as shown in Table 2, a slightly lower prevalence was recorded for joint pains, CTS, and bone cysts in the AN69 cases, no statistically significant differences 
were obtained, whether patients were dialysed for less, or more, than 2 years with cellulosic membranes before switching to AN69.

The distribution of age groups is given for combined groups of cases and controls as mentioned in Table 2 and for specific symptoms of dialysis-associated arthropathy in Fig. 3. $\chi$-square analysis shows a significant relationship between age and joint pains $(P<0.01)$. For the other symptoms of dialysis-related osteoarthropathy $\chi$-square analysis did not show a significant relationship with age (carpal-tunnel syndrome, $P>0.20$; cysts, $P>0.20$; erosive arthropathy, $P>0.95$ ). $\beta_{2}$-Microglobulin values were given for only a minority of patients and thus it was not possible to make a meaningful comparison between cases and controls for this variable. A more detailed analysis, using Haldane's test, shows the proportional contribution of age for those with and without joint pains (Fig. 4). This test confirms a statistically significant relationship $(P<0.04)$. There was no difference in the median and mean time on dialysis for patients with and without joint pains (median: 10 years for both groups).

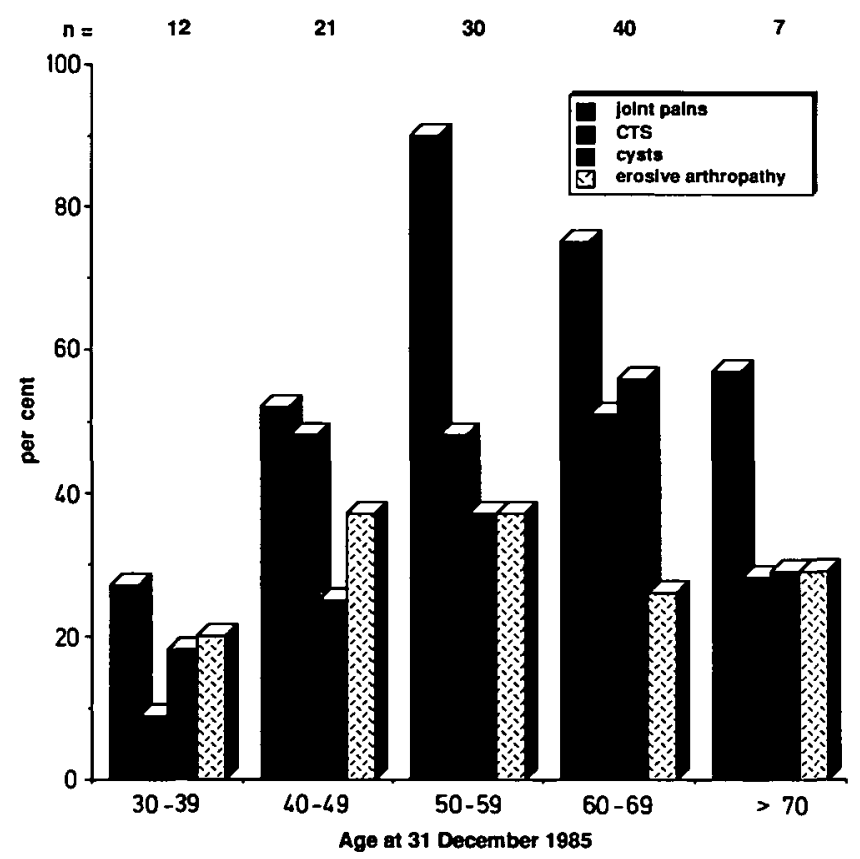

Fig. 3. Prevalence of signs and symptoms of dialysis associated osteoarthropathy according to age at 31 December 1985 for all cases and controls.

\section{Discussion}

Dialysis arthropathy or haemodialysis-associated amyloidosis contributes to disability in dialysis patients. This painful bone and joint disease has been attributed by many investigators to the formation of amyloid deposits

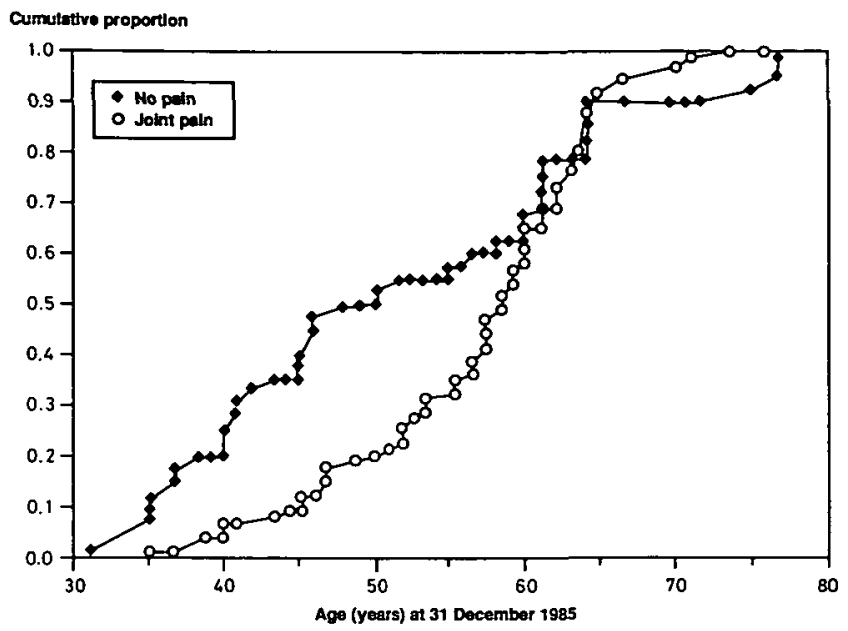

Fig. 4. A cumulative proportion with age of dialysis patients with and without joint pains according to their ages on 31 December 1985.

consisting mainly of $\beta_{2}$-microglobulin $[5,6]$. However, other causative mechanisms have been proposed, such as deposits of haemosiderin [9]

It is beyond doubt that blood concentrations of $\beta_{2}$ microglobulin are greatly elevated in haemodialysis patients and that these concentrations can be influenced by the type of membrane used for haemodialysis. The use of more porous membranes, such as AN69, yield a $50 \%$ reduction in blood concentrations in comparison with cuprophane membranes. However, even with the use of more porous dialysis membranes, blood concentrations for $\beta_{2}$-microglobulin can be as much as 20 -fold the normal values [8].

It has been reported that the number of cases of arthropathy in patients dialysed with AN69 membranes was smaller than in patients treated with cuprophane membranes. However, no rigorously comparable controls were available and patients were not evaluated for grafting, type of renal disease and sex [7]. In view of the fact that only 55 pairs of patients within the EDTA Registry database fulfilled the criteria of this case control study, and no more than 27 cases had less than 2 years of cellulosic membrane dialysis, it is not surprising that no properly controlled data are available.

Some conclusions can be drawn from this study despite its shortcomings such as its multicentre character, the small number of patients and, as yet, a poorly defined syndrome. More than $60 \%$ of all the patients reported in this study, and who had been dialysed for at least 9 years, suffered from painful osteoarticular disease. Although the severity of the disease and the time of onset of symptoms were not evaluated, of all signs and symptoms the prevalence of joint pains differed between age groups (Figs 3,4). However, more data are needed to validate these findings.

The 27 AN69 cases who had received initial treatment with cellulosic membranes for less than 2 years tended to 
have a lower prevalence of some signs and symptoms of $\beta_{2}$-microglobulin amyloidosis than the 27 controls (Fig. 1). However, differences in prevalence did not reach statistical significance. The 28 cases with more than two years' dialysis solely on cellulosic membranes before they went on to AN69 dialysis showed minor and inconsistent differences compared to the control group on cuprophane alone (Fig. 2).

The use of different membranes for haemodialysis is influenced by doctors' desire to eliminate uraemic toxins, prevent acute side-effects and reduce long-term morbidity. If a clear clinical advantage can be established for one type of membrane then this will be chosen irrespective of its cost. One of the most important longterm sequelae of haemodialysis seems to be dialysis osteoarthropathy (or dialysis-associated amyloidosis). However, in this retrospective case control study it could not be proven that patients dialysed for more than 10 years with cuprophane, a dialysis membrane with low porosity, would suffer more frequently from this disease than those dialysed predominantly with the highly porous polyacrylonitrile (AN69) membrane. Of the symptoms characterising this condition, joint pains were found more frequently in older patients. This suggests a link with age which is worthy of a more detailed study.

Acknowledgements. The work of the EDTA Registry has been supported by grants from Governments and National Societies of Nephrology.
Grants have also been made by the AKZO Group, Asahi Medical Co. Ltd, B. Braun Melsungen AG, Baxter Healthcare Corporation, Bellco $\mathrm{SpA}, \mathrm{CD}$ Medical International Ltd, Fresenius Ltd, Gambro AB Hospal Ltd, and Sandoz AG.

We thank all those doctors and their staff who have kindly completed EDTA Registry questionnaires, thus making the study possible.

\section{References}

1. Kachel HG, Altmeyer P, Baldamus CA, Koch KM. Deposition of an amyloid like substance as a possible complication of regular dialysis treatment. Contrib Nephrol 1983; 36: 127-132

2. Laurent G, Calemard E, Charra B. Dialysis related amyloidosis. Kidney Int 1988; 33 [Suppl 24]: S32-34

3. Brown EA, Arnoco IR, Gower PE. Dialysis arthropathy: complication of long-term treatment with haemodialysis. Br Med J 1986; 292: 163-166

4. Schwarz A, Keller F, Syfert S, Poll W, Molzahn M, Distler A Carpal tunnel syndrome: a major complication in long-term haemodialysis patients. Clin Nephrol 1984; 22: 133-137

5. Baroin T, Kuntz D, Zingraff J, Voisin MC, Zelmar A, Lansaman J. Synovial amyloidosis in patients undergoing long-term hemodialysis. Arthritis Rheum 1985; 28: 1052-1058

6. Shirahama T, Skinner M, Cohen AS et al. Histochemical and immunohistochemical characterization of amyloid associated with chronic hemodialysis as beta-2-microglobulin. Lab Invest 1985; 53: 705-709

7., van Ypersele de Strihou C, Hinhon B, Vandenbrouke JM. Dialysis amyloidosis. Adv Nephrol 1988; 17: 401-422

8. Caverle Y, Simon P, Ang KS, Cam G, Catheline M. Serum beta2-microglobulin levels in hemodialysed uremics depending upon permeability of dialysis membranes. Kidney Int 1987; 31: 229

9. Sethi D, Woodrow DF, Brown EA et al. Beta-2-microglobulin derived amyloid and iron in dialysis arthropathy. Nephrol Dial Transplant 1987; 2: 449 